\title{
Identification of colorant materials after an artificially aged process
}

\section{Identificación de materiales colorantes tras un proceso de envejecimiento artificial}

\author{
Ana López-Montes*1, Anne-Laurence Dupont², Natalia Tello Burgos ${ }^{1}$, Teresa Espejo ${ }^{1}$, \\ Rosario Blanc ${ }^{3}$ \\ 1. Dpto. Pintura. Facultad de Bellas Artes. Ed. Aynadamar, Avd. Andalucía s/n. 18071 Granada, España.
}

2. Centre de recherche sur la conservation des collections, (CRC, USR 3224), Sorbonne Universités, Ministère de la Culture et de la Communication , Muséum National d'Histoire Naturelle, CNRS, CP 21, 36 rue GeoffroySaint-Hilaire, 75005 Paris, Francia.

3. Dpto. Química Analítica. Facultad de Ciencias. Avd. Fuentenueva s/n. 18071 Granada, España.

(*) E-mail: alopezmontes@ugr.es

Received: 08/04/2016 Accepted: 01/07/2016

DOI: 10.7149/OPA.49.3.49006

\begin{abstract}
:
Chemical composition and physical characteristics of art materials (support, paint layer, protection layer...) depend on different factors such as their origin, the application process, the storage conditions throughout the centuries and the ageing processes. The identification of aged organic dyes can be difficult due to the possible changes that their physical and chemical characteristics could have undergone with time. A comparative study between standard samples and samples aged in a controlled climate chamber under drastic conditions of humidity, temperature and light was carried out. The techniques of identification and evaluation used were High Performance Liquid Chromatography (HPLC) and UV-visible spectroscopy. Results showed that the cellulosic fibers confer greater resistance to dyes in all cases.
\end{abstract}

Key words: Colour in art, identification, natural dyes, artificial aged process, conservation.

\section{RESUMEN:}

La composición química y las características físicas de los materiales artísticos (soporte, capa pictórica, capa de protección...) dependen de diferentes factores tales como la fuente de origen, el procedimiento utilizado para su aplicación, las condiciones de almacenamiento a través del tiempo y el proceso de envejecimiento. Se destaca, en este punto, la problemática de la identificación de los colorantes orgánicos después de su envejecimiento, ya que, en muchos casos, pueden haberse modificado sus características físicas o químicas. Para la identificación de los colorantes bajo estas condiciones se realizó un estudio comparativo entre las muestras patrón sin envejecer y las muestras artificialmente envejecidas en una cámara climática, sometiéndolas a condiciones drásticas de humedad, temperatura e iluminación. Las técnicas que se emplearon para identificar y evaluar los cambios sufridos por estos colorantes fueron la cromatografía líquida de alta resolución (HPLC) y la espectroscopía UV-visible. Los resultados demostraron que las fibras celulósicas confieren mayor resistencia a los colorantes en todos los casos.

Palabras clave: Color en arte, identificación, colorantes naturales, proceso de envejecimiento artificial, conservación. 


\section{REFERENCES AND LINKS / REFERENCIAS Y ENLACES}

[1] J. Hutchings, "Colour in folklore and tradition - The Principles", Color Res Appl 29, 57-66 (2004). http://dx.doi.org/10.1002/col.10212

[2] D. Bomford, The History of colour in Art, in Color: Art \& Science, T. Lqmb, J. Borriau Edts., Cambridge University Press (1995).

[3] M.S. García-Falcón, J. Simal-Gámdara, "Determination of food dyes in soft drinks containing natural pigments by liquid chromatography with minimal clean-up", Food Control 16, 293-297 (2005). http://dx.doi.org/10.1016/j.foodcont.2004.03.009

[4] J.F. Senackerib, Colorants for Non-Textile Aplication, H.S. Freeman and A.T. Peters ed., Carolina, USA (2000).

[5] N. Chigurupati, L. Saiki, C. Gayser, A.K. Dash, "Evaluation of red cabbage dye as a potential natural color for pharmaceutical use", Int J Pharm 241, 293-299 (2002). http://dx.doi.org/10.1016/S0378-5173(02)00246-6

[6] C. Millani, A. Romani, G. Favaro, "A spectrophotometric and fluorimetric study of sime anthraquinoid and indigoid colorants used in artistic paintings", Spectroc Acta Part A: Molecular and Biomolecular Spectroscopy 54, 581-588 (1998). http://dx.doi.org/10.1016/S1386-1425(97)00240-0

[7] D. Buti, D.Domenici, D. Miliani, T. García Sáiz, T.C. Gómoz Esponoza, F. Jimenez Villalba, A. Verde Casanova, A. Sabía de la Mata, A. Romani, F. Presciutti, B. Doherty, B.G. Brunetti, A. Sgamellotti, "Noninvasive investigation of a pre-Hispanic Maya screenfold book: the Madrid Codex", J. Archaeol Sci 42, 166-178 (2014). http://dx.doi.org/10.1016/i.jas.2013.08.008

[8] J. Seixas de Melo, A.P. Moura, M.J. Melo, "Photophysical and Spectroscopic Studies of Indigo Derivatives in Their Keto and Leuco Forms", J Physical Chem A 108, 6975-6981 (2004). http://dx.doi.org/10.1021/jp049076y

[9] A. López-Montes, T. Espejo, J.L. Vílchez, R. Blanc García "Notas sobre la identificación madiante técnicas de análisis químico del amarillo de azafrán envejecido artificialmente", Opt Pura Apl 41, 261-265 (2008).

[10] A. López-Montes, C. Plata Rios, R. Blanc, J.L. Vílchez, T. Espejo "Estudio de los cambios inducidos por tratamientos de desacidificación en documentos gráficos", 16th International Meeting on Heritage Conservation, (2006) 537-549.

[11] E. Kampasakali, E.A. Varella, "The Russian avant-garde painting palette: Documentary and phisicochemical codification of organic colorants", J Cult Herit 9, 77-88 (2008). http://dx.doi.org/10.1016/i.culher.2007.02.005

[12] C.Clamenti, W. Nowik, A. Romani, F. Cibin, G. Favaro "A spectrometric and chromatographic approach to the study of ageing of madder (Rubia tinctorum L.) dyestuff on wool", Anal Chim Acta 596, 46-54 (2007). http://dx.doi.org/10.1016/j.aca.2007.05.036

[13] I. Degano, M. Biesaga, M.P. Colombini, M. Trojanowicz, "Historical and archaeological textiles: an insight on degradation products of wool and silk yarns", J Chromatogr A 1218, 5837-5847 (2011). http://dx.doi.org/10.1016/i.chroma.2011.06.095

[14] K. Selim, M. Tsimidou, C.G. Biliaderis, "Kinetic studies of degradation of saffron carotenoids encapsulated in amorphous polymer matrices", Food Chem 71, 199-206 (2000). http://dx.doi.org/10.1016/S0308-8146(00)00156-4

[15] R. Rondao, J.S. Seixas de Melo, D. Vasco, B. Bonifacio, M.J.J., Melo, "Dehydroindigo, the Forgotten Indigo and Its Contribution to the Color of Maya Blue", Phys Chem A 114, 1699-1708 (2010). http://dx.doi.org/10.1021/jp907718k

[16] R. Blanc, T. Espejo, A. López-Montes, D. Torres, G. Crovetto, A. Navalón, J.L. Vílchez, "Sampling and identification of natural dyes in historical maps and drawings by liquid chromatography with diodearray detection", J Chromatogr A 1122, 105-113 (2006).

http://dx.doi.org/10.1016/j.chroma.2006.04.056

[17] C. Ahn, S.K. Obendorf, "Dyes on archaeological textiles: Analyzing alizarin and its degradation products", Text Res J 74, 949-954 (2004). http://dx.doi.org/10.1177/004051750407401102 
[18] F. Gosetti, U. Chiuminatto, U. Mazzucco, R. Mastroianni, E. Marengo, "Ultra-High-performance liquid chromatography/tandem high-resolution mass spectrometry analysis of sixteen red beverages containing carminic acid: identification of degradation products by using principal component analysis/discriminant analysis", Food Chem 167, 454-462 (2015).

http://dx.doi.org/10.1016/i.foodchem.2014.07.026

\section{Introducción}

Para poder reconstruir el pasado, entender las culturas, interpretar su simbología... es necesario intentar recuperar, de la manera más fiel posible, su riqueza cromática, haciendo un estudio exhaustivo del color [1]. Una recreación realista de los colores del pasado se ve enormemente dificultada porque el paso del tiempo los ha modificado de manera extraordinaria [2]. El envejecimiento natural, las condiciones ambientales y la mano del hombre, han hecho que, a menudo, estos cambios o pérdidas sean irreparables.

Durante la revisión bibliográfica se acreditó la abundancia de publicaciones donde se recogen estudios sobre colorantes orgánicos naturales de origen animal o vegetal, debido a su interés en la industria cosmética, farmacéutica y alimentaria [3-5]. También se han encontrado trabajos sobre identificación de colorantes empleados como capas pictóricas [6,7] o como tintes para textiles. La mayoría de ellos se centraban en la identificación y caracterización de materiales [8].

En los últimos años ha crecido el interés por la valoración de las alteraciones que pueden sufrir estos materiales por efecto de agentes externos o por el propio envejecimiento natural. En la última década aparecen los primeros estudios sobre la influencia de los factores ambientales en colorantes naturales [9], estudios relativos al envejecimiento acelerado de colorantes usados en documentos gráficos [10,11], donde se describen las variaciones tonales producidas, envejecimiento de sustancias rojas empleadas como tintes en textiles [12,13], degradación de los carotenoides del azafrán [14], y alteraciones de la molécula de índigo, independientemente de su aplicación [15].

Debido a la escasez de publicaciones en comparación con la magnitud de este campo, se ha querido abordar esta investigación cuyo objetivo principal es evaluar los cambios ópticos y químicos de los colorantes simulando los procesos de envejecimiento natural para ayudar a su identificación en obras de arte y saber si están presentes a pesar de las alteraciones que hayan podido sufrir.

El estudio se ha realizado sometiendo al rojo carmín, rojo de rubia, amarillo de goma guta y azul de índigo, a un proceso de envejecimiento acelerado, en disolución (como elemento independiente) y aplicados sobre papel (como material colorante). Se han valorado las variaciones de color por espectrometría UVvis, y las modificaciones en la composición por cromatografía líquida de alta resolución acoplada a un detector de diodos en línea (HPLC-PDA).

\section{Materiales y métodos}

\section{2.a. Materiales, reactivos y disolventes}

Se ha escogido un papel de composición conforme a la de muchos papeles medievales, de fibra de yute de factura artesanal sin adición de encolantes ni colorantes, elaborado por el molino papelero de Capellades.

Los colorantes naturales fueron obtenidos de diferentes fuentes: el rojo de carmín fue extraído del insecto Coccus cacti, el rojo de rubia de la raíz de la planta Rubia tinctorum, el amarillo de goma guta de la resina del árbol Garcinia hanburü y el azul de índigo de las hojas prensadas de la planta Indigofera tinctoria. Todos ellos fueron suministrados por Kremer-Pigmente (Cracovia, Polonia).

El reactivo dodecil sulfato sódico (SDS) fue suministrado por Sigma Aldrich Chemie (Steinheim, Alemania).

El agua empleada, en todos los casos, fue purificada mediante el sistema Milli-Q (Millipore, Bedford, USA).

Los disolventes metanol, ácido acético y ácido trifluoroacético (99\% pureza) fueron proporcionados por Merck (Darmstadt, Alemania); el acetonitrilo fue proporcionado por Panreac (Barcelona, España) y todos ellos fueron de calidad gradiente-HPLC. 


\section{2.b. Instrumentación y programas informáticos}

Los espectros de absorbancia se realizaron con un equipo Agilent 8453E (Waldbronn, Alemania) realizando el tratamiento de datos con el programa Chemstation.

El equipo cromatográfico fue Agilent 1100 series HPLC (Agilent Technologies, Palo Alto, USA) equipado con una bomba cuaternaria, un desgasificador, muestreador automático, inyección automática, un compartimento de termostatización de la columna y un detector de diodos acoplado en línea. La separación cromatográfica se llevó a cabo con una columna Luna NH2 100a (250mm x 4,6 mm d.i., $5 \mu \mathrm{m}$ partícula) de Phenomenex (Torrance, USA). La columna se protegió con una precolumna $\mathrm{NH}_{2}$ aminoaminopropileno (Phenomenex) de $4 \mathrm{~mm}$ x $3 \mathrm{~mm}$ d.i. El cromatógrafo fue controlado a través del programa ChemStation para LC 3D (Agilent).

La cámara de envejecimiento utilizada fue Solarbox 3000e RH (Italia) equipada con una lámpara de xenón (irradiancia 300-800 $\mathrm{nm}$ ) y filtro indoor S208/S408. El programa empleado para el control de las condiciones fue Xen43.

\section{2.c. Preparación de las muestras Extracción de los colorantes}

La preparación de las muestras desde su origen natural se realizó de acuerdo a los parámetros optimizados en trabajos anteriores por este mismo equipo de trabajo [16].

Para extraer el rojo de carmín se colocaron 10 insectos en un vaso de precipitado con 50 ml de agua calidad Milli-Q a $30^{\circ} \mathrm{C}$ durante $15 \mathrm{~min}$. Después, la disolución coloreada fue filtrada con papel de filtro neutro.

El rojo de Rubia se extrajo de la raíz de Rubia tinctorum L. triturada colocando 3 gr en un vaso de precipitado con $50 \mathrm{ml}$ de agua calidad Milli-Q a 300 durante $15 \mathrm{~min}$. La disolución coloreada fue filtrada con papel de filtro neutro.

El amarillo de goma guta se preparó colocando 1 gr de resina en un vaso de precipitado con $50 \mathrm{ml}$ de metanol en un baño de ultrasonidos durante $20 \mathrm{~min}$. La disolución coloreada fue filtrada con papel de filtro neutro.

Para obtener el azul índigo, se tomaron 0,2 gr de las hojas prensadas de la planta Indigofera tinctoria y se colocaron en un vaso de precipitado con $50 \mathrm{ml}$ de ácido acético en una concentración de 17,5 M en un baño de ultrasonidos durante $45 \mathrm{~min}$. La disolución fue filtrada con un filtro de nylon de 0,2 $\mu$ m de tamaño de poro.

\section{Preparación de las muestras para el envejecimiento artificial}

Se prepararon dos grupos de muestras: Grupo I compuesto por los colorantes aplicados sobre papel, y Grupo II de colorantes en disolución (Fig. 1).

Al Grupo I se aplicó una capa de color homogénea sobre el papel con pincel. Para el Grupo II se prepararon viales de vidrio transparente que contenían $5 \mathrm{ml}$ de cada una de las disoluciones previamente preparadas como se indica en el apartado anterior: rojo de carmín y rojo de rubia disueltos en agua, el amarillo de goma guta en metanol y el azul de índigo en ácido acético.

Las muestras se introdujeron en la cámara de envejecimiento en las condiciones adecuadas para el estudio (muestras LUZ) y una réplica de cada una de las muestras de ambos grupos se mantuvo en un espacio estanco en condiciones estables de humedad y temperatura a $25^{\circ} \mathrm{C}$ y $30 \%$ de Humedad Relativa $\left(\mathrm{H}_{\mathrm{R}}\right)$ y protegida de la luz, que se emplearía como muestra de referencia y control (muestras REF). Las condiciones de envejecimiento responden a las normas ISO 5630-3(1996) e ISO 1134-1(2004).

\section{Toma y tratamiento de muestra para análisis}

Las muestras del Grupo I se tomaron con la ayuda de un pincel humedecido con SDS 0,1 M frotando suavemente sobre una superficie de $1 \mathrm{~cm}^{2}$, introduciéndolas en un micro-vial al que se añadió $100 \mu \mathrm{l}$ de SDS 0,1 M en disolución acuosa. Para las muestras del Grupo II se tomó un volumen de 3 ml y se introdujo en la cubeta de cuarzo para ser analizada. 


\section{2.d. Metodología analítica aplicada}

Las muestras del Grupo I se analizaron por HPLC-DAD y los datos que se emplearon para la identificación de los colorantes fueron el tiempo de retención $\left(t_{R}\right)$ y el espectro UV-vis obtenido en los máximos de los picos cromatográficos.

Se recurrió a la preparación de las muestras del Grupo II para su estudio por espectrofotometría con el fin de caracterizar e identificar los espectros correspondientes a los distintos colorantes, y para estudiar la evolución del envejecimiento de estos colorantes independientemente del soporte al que se encuentraban fijados.

\section{Condiciones del envejecimiento artificial acelerado}

Las condiciones respondieron a las normas ISO 5630-3:1996 e ISO 11341:2004, que fueron 80ํㅡ, $65 \% \mathrm{H}_{\mathrm{R}}$ e irradiancia de $550 \mathrm{Wm}^{-2}$ con filtro para eliminar la radiación ultravioleta (filtro indoor). Los tramos de envejecimiento fueron de $0 \mathrm{~h}, 24 \mathrm{~h}, 48 \mathrm{~h}, 72 \mathrm{~h}$ y $144 \mathrm{~h}$.

\section{Espectrofotometría UV-vis}

Después de cada sesión de envejecimiento se extrajeron $3 \mathrm{ml}$ de las disoluciones del Grupo II para introducirlos en la cubeta de cuarzo e identificar los distintos colorantes. El estudio de absorción se realizó en un rango comprendido entre 190 y $1100 \mathrm{~nm}$. Se realizaron 3 réplicas instrumentales y 3 réplicas experimentales de cada condición.

\section{Cromatografía líquida de alta resolución}

La metodología analítica aplicada para la identificación de los distintos colorantes mediante HPLC fue desarrollada con anterioridad [16]. Los parámetros cromatográficos fueron los siguientes:

La fase móvil consistió en una disolución de SDS $40 \mathrm{mM}$ con tampón fosfato $10 \mathrm{mM}$ (pH 2.3) y 0.1\% de ácido trifluoroacético como eluyente A. Como eluyente B se usó acetonitrilo con un programa gradiente de $5 \%$ de B a $95 \%$ de B en $45 \mathrm{~min}$ frente al diluyente A. El flujo fue de 0,6 ml/min hasta el min $40 \mathrm{y}$ aumentando a $1 \mathrm{ml} / \mathrm{min}$ hasta el final de la separación. La columna se mantuvo a $45^{\circ} \mathrm{C}$ y el volumen de inyección fue de $20 \mu$ l. La detección se llevó a cabo con un detector de diodos (PDA) instalado en el equipo, ofreciendo información espectral en pasos de $2 \mathrm{~nm}$ entre 190 y $900 \mathrm{~nm}$ durante todo el análisis cromatográfico.

\section{Resultados y discusión}

La apariencia de las muestras de los Grupos I y II durante el transcurso del proceso de envejecimiento pueden observarse en la figura 1.

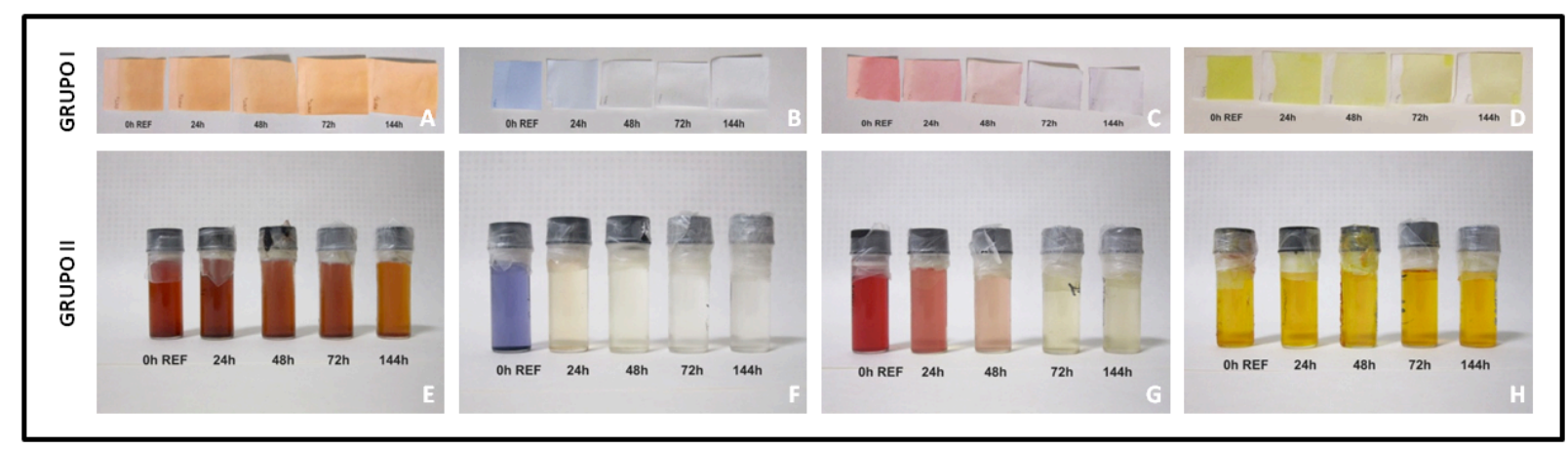

Fig.1. Evolución del proceso de envejecimiento de las muestras de Grupo I y II . A y E: Rojo de Rubia; B y F: Azul de índigo; C y G: Rojo de carmín; D y H: Amarillo de goma guta.

La estructura molecular de cada uno de los componentes principales de los colorantes objeto de estudio puede consultarse en la figura 2 . 


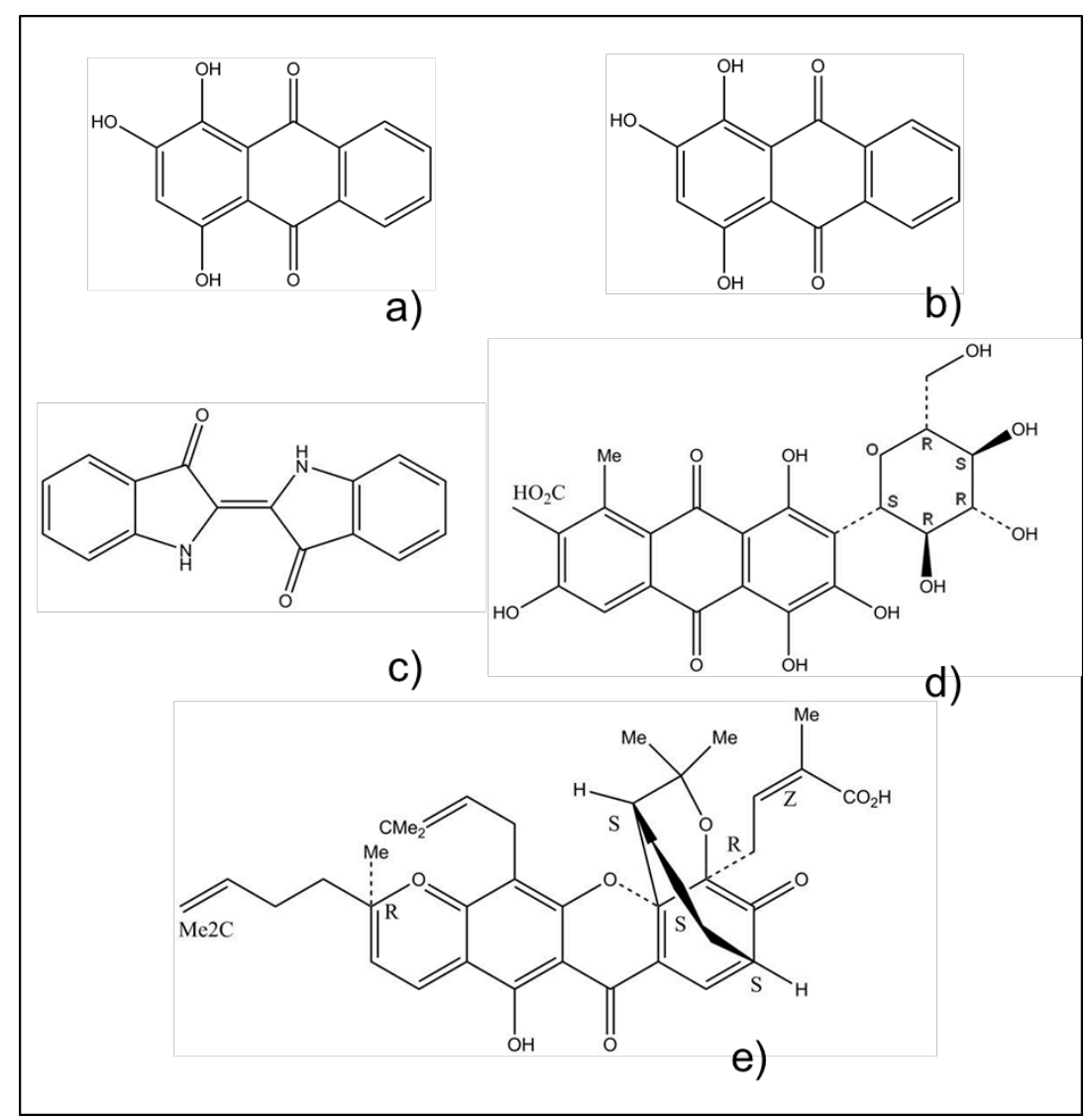

Fig.2. Componentes principales de los colorantes. Rojo de Rubia: a) molécula de alizarín y b) molécula de purpurín; azul de índigo: c) índigo; rojo de carmín: d) ácido carmínico; Amarillo de goma guta: d) ácido gamboico.

\section{3.a. Rojo de Rubia}

La tonalidad del rojo procedente de la raíz de Rubia tinctorum L. resistió sin modificaciones aparentes hasta el final de proceso de envejecimiento en las muestras del Grupo I y II matizándose con un viraje hacia el amarillo. Las muestras de referencia de los Grupos I y II mantuvieron la tonalidad original.

Durante el estudio del Grupo I por HPLC, se observó que la muestra de referencia a 0h presentaba 2 picos cromatográficos correspondientes a alizarín $\left(t_{R}: 33,50 \mathrm{~min}\right.$ ) y purpurín ( $t_{\mathrm{R}}: 36,28 \mathrm{~min}$ ), los dos componentes principales del colorante obtenidos de la raíz de Rubia (compuestos definido previamente por el mismo equipo investigador [16]) (Fig. 2 y 3). A partir de la primera sesión de envejecimiento y hasta las $72 \mathrm{~h}$, estas señales fueron disminuyendo en área y altura, llegando a ser completamente imposibles de identificar a $144 \mathrm{~h}$.

El espectro UV-vis obtenido de las muestras del Grupo II (Fig. 3), mostró idénticos resultados, perdiendo intensidad de absorción respecto al espectro de referencia $(201,236,280$ y $510 \mathrm{~nm}$ ). A partir de las $72 \mathrm{~h}$ se observó un desplazamiento de la banda de absorción de $510 \mathrm{~nm}$ a $430 \mathrm{~nm}$, lo que indica una tendencia de la tonalidad roja hacia el amarillo.

Según C. Clementi et col. [12] el purpurín es el principal componente de esta planta, responsable de las propiedades cromáticas del rojo de Rubia y, por lo tanto, de su espectro de absorbancia. También lo hace responsable de la alteración de este rojo, siendo la degradación fotoquímica, debido a la exposición a la luz, el factor más importante. En sus investigaciones marca las variaciones obtenidas en los espectros de absorbancia y los estudios cromatográficos pero no identifica la presencia de nuevos compuestos ni el proceso químico de la degradación de alizarín y purpurín como ha ocurrido en este caso. 


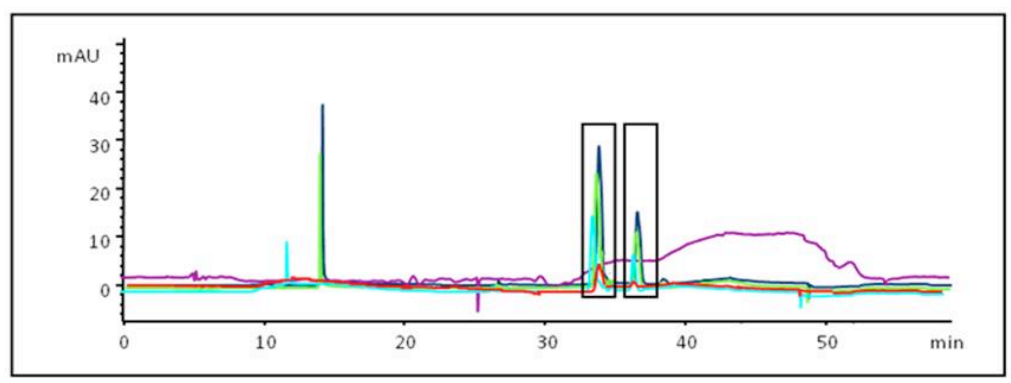

Cromatograma raiz Rubia Tinctoria

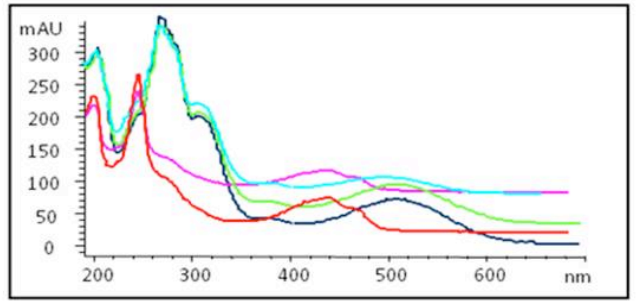

Espectro UV-vis raiz Rubia TInctoria

Fig. 3. Cromatogramas y espectros UV-vis de la serie envejecida de rojo de Rubia.

I. Degano et col. [13] presentan una lista de productos secundarios encontrados en muestras históricas de textil envejecidas de manera natural que podrían ser atribuidas al deterioro del rojo de Rubia y a su combinación con otros productos presentes. Esta hipótesis no la confirma, ya que remarca la contraposición entre los resultados obtenidos por C. Clementi, quien no identifican ningún producto de degradación, y los ensayos realizados por Ahn y Obendorf [17], que indica la posible presencia de ácido ftálico, anhidro ftálico y ácido benzoico como posibles productos de degradación.

\section{3.b. Azul de índigo}

En las muestras del Grupo I se observó que la tonalidad azulada se desvaneció considerablemente rápidamente a partir de la primera sesión de envejecimiento desapareciendo a partir de la segunda sesión. El colorante en disolución del Grupo II se comportó de manera similar, virando completamente hacia el amarillo en la primera sesión, y volviéndose transparente a partir de la segunda sesión de envejecimiento (Fig. 1).

Al analizar las muestras del Grupo I por HPLC, se observó que la señal de índigo era reconocible hasta las $24 \mathrm{~h}$ de envejecimiento ( $\mathrm{t}_{\mathrm{R}}: 34,77 \mathrm{~min}$ ) [16]. Durante las $24 \mathrm{~h}$ de envejecimiento, apareció un segundo pico cromatográfico (a) con $t_{\mathrm{R}}$ : 24,66 min que fue desapareciendo, a la vez que la señal de índigo, a partir de las $72 \mathrm{~h}$ de envejecimiento (Fig. 4).

En el Grupo II, la muestra de referencia de azul de índigo en disolución sin envejecer presentaba bandas de absorción características a 286, 348 y $623 \mathrm{~nm}$ [16]. Las muestras envejecidas se volvieron de color amarillo pasadas $24 \mathrm{~h}$, y sus espectros de absorbancia presentaron tres nuevas bandas de absorción a 250, 307 y $380 \mathrm{~nm}$, perdiéndose las banda a 348 y 623nm (Fig. 4).

Las muestras que se mantuvieron en condiciones estables de humedad y temperatura, y protegidas de las radiaciones luminosas (muestras REF), también presentaron el mismo comportamiento tanto en el estudio por HPLC como por espectrofotometría, aunque de manera más pausada que las muestras envejecidas artificialmente, cuyas variaciones fueron más rápidas y acentuadas. Este resultado llevó a pensar que esta transformación no se debía únicamente a la exposición a temperatura, iluminación y humedad relativa elevados, sino que estos fueron parámetros aceleradores de las reacciones de degradación. 


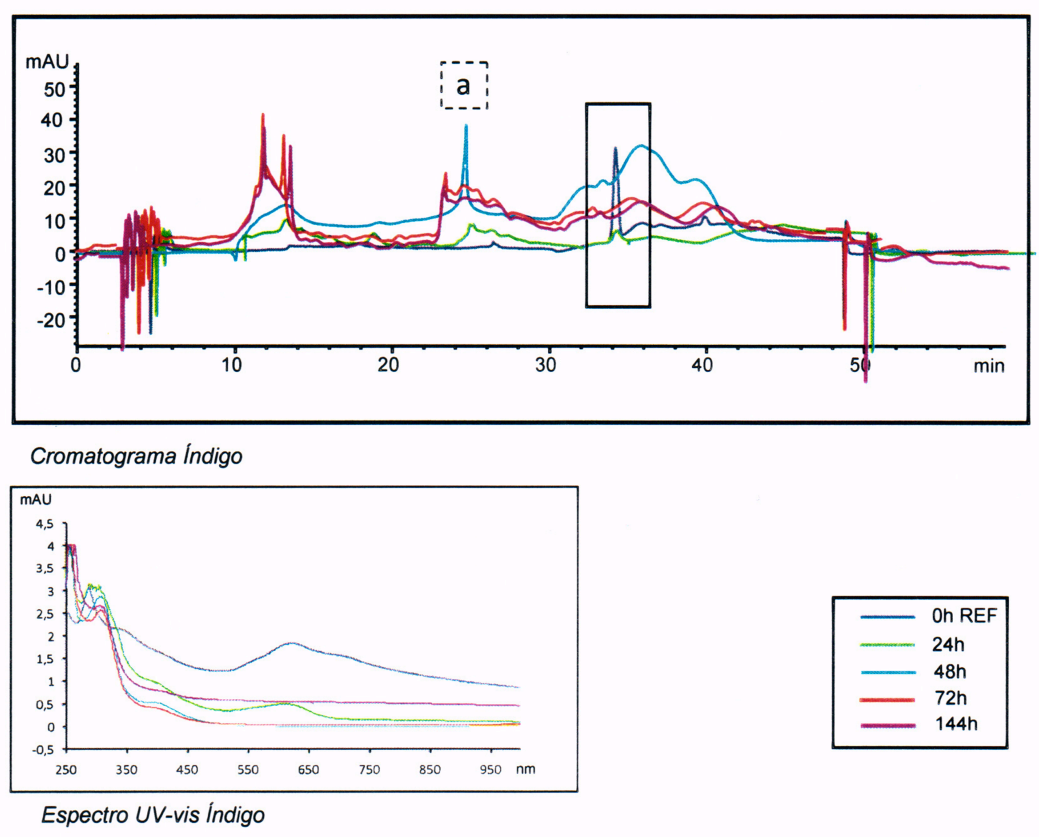

Fig. 4. Cromatogramas y espectros UV-vis de la serie envejecida de azul índigo.

La consulta bibliográfica sobre la alteración de la molécula indicó que esta molécula se transforma, por oxidación, en dehidroíndigo [15].

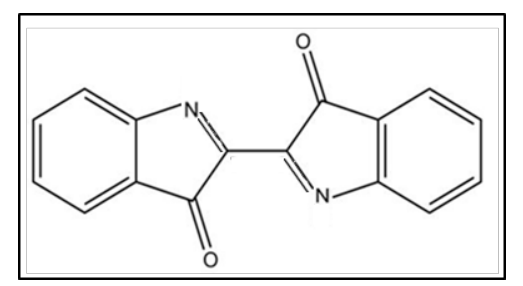

Fig.5. Molécula de dehidroíndigo.

Se deduce, por tanto, que el cromatograma obtenido de la muestra inicial a 0h se correspondía con las moléculas de índigo. La formación de la molécula de dehidroíndigo fue evidente en las muestras envejecidas durante 24 y $72 \mathrm{~h}$. A partir de ese momento la molécula de dehidroíndigo también pudo alterarse. En el estudio por espectrofotometría, se observó la existencia de una nueva molécula que podría ser la de dehidroíndigo, ya que las bandas de absorción coincidieron a 348 y $600 \mathrm{~nm}$ como indican Rondao et col. [15].

\section{3.c. Rojo de carmín}

La tonalidad roja sobre papel fue apreciable hasta las $48 \mathrm{~h}$, haciéndose muy tenue, aunque presente, en los dos últimos tramos de envejecimiento. La disolución coloreada se volvió bastante transparente, con tendencia al rosa a las $24 \mathrm{~h}, \mathrm{y}$ al amarillo a partir de las $48 \mathrm{~h}$, llegando en estas condiciones hasta el final del proceso (Fig. 1). Las muestras de referencia de los Grupos I y II mantuvieron la tonalidad original.

En la valoración de los resultados mediante HPLC de las muestras del Grupo I (Fig. 6), se observó que el cromatograma de la muestra de referencia $(0 \mathrm{~h}$, sin envejecer) presentaba un pico cromatográfico que corresponde al ácido carmínico (Fig. 2), con $\mathrm{t}_{\mathrm{R}}$ : 14,44 min [16]. Tras la primera sesión de envejecimiento (24h), ese pico cromatográfico se adelantó 2 min y apareció un nuevo pico (a) con $t_{R}: 23,77$ min. Después de la segunda sesión de envejecimiento (48h), el pico correspondiente al ácido carmínico se mantuvo, y aumentó el área y altura de la nueva señal que apareció a los 23,77 min, lo que confirmaría la aparición de un nuevo compuesto. Este nuevo compuesto con un $t_{R}$ mayor indica la formación de una molécula con mayor factor de retención que el ácido carmínico, por tanto menos polar debido, quizá, a una oxidación. A 
partir de ese tramo de envejecimiento y hasta el final se sigue observando claramente el pico correspondiente al ácido carmínico y el del segundo nuevo compuesto. Por lo tanto, el cromatograma conserva el pico cromatográfico correspondiente al ácido carmínico hasta el final del proceso de envejecimiento, junto con otro compuesto, señalados a partir de las $24 \mathrm{~h}$ de exposición.

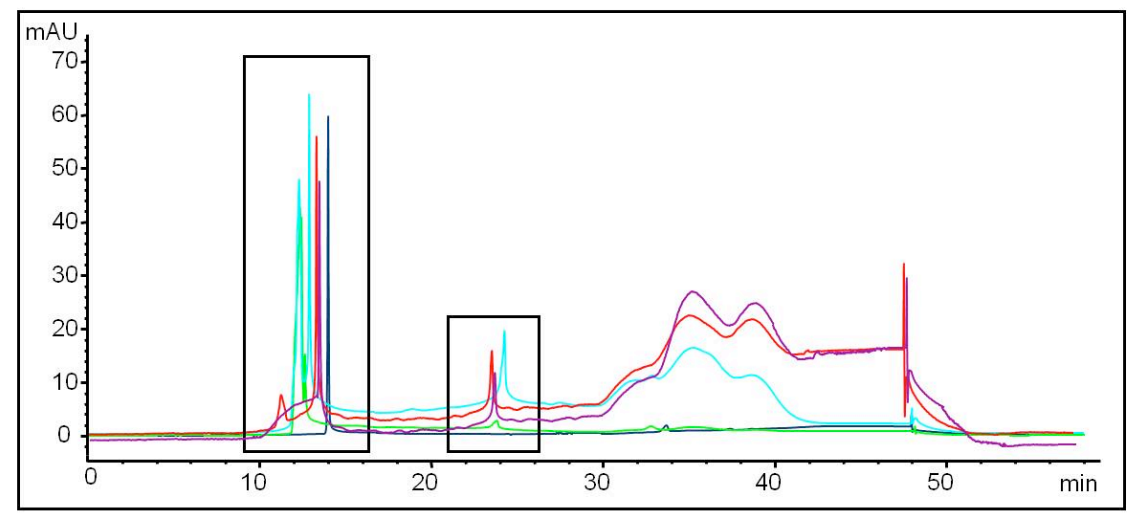

Cromatograma Carmín

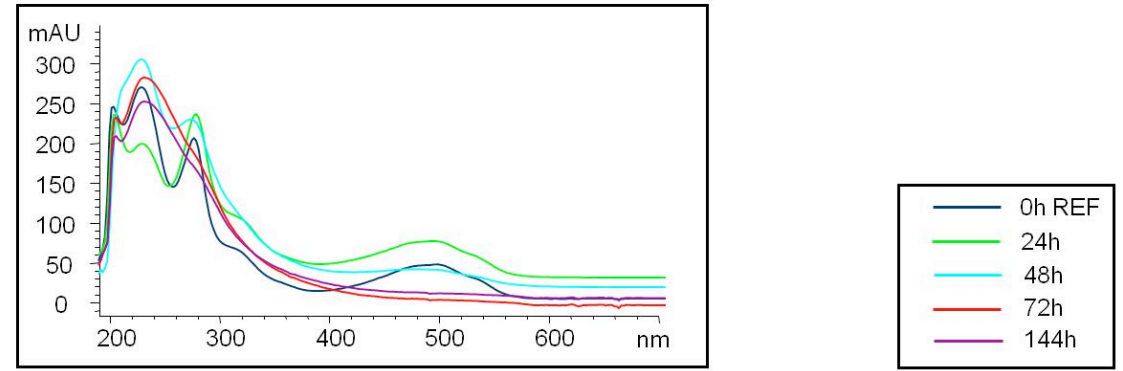

Espectro UV-vis Carmín

Fig. 6. Cromatogramas y espectros UV-vis de la serie envejecida de rojo de carmín.

En la valoración de los resultados por espectrometría UV-vis de las muestras del Grupo II (Fig. 6), se observó que el espectro se fue modificando a cada paso, suavizándose la línea y desapareciendo la curva cercana a longitudes de onda propias del rojo (cercanas a los 500nm) a partir de las $48 \mathrm{~h}$ de exposición. El espectro de absorción de la muestra inicial de referencia a 0h presentó bandas de absorción a 206, 234, 284, 334, 521 y $563 \mathrm{~nm}$, propias de rojo de carmín [16]. Tras el primer tramo de envejecimiento, el espectro fue similar, disminuyendo la intensidad de las 2 primeras bandas, aumentando la intensidad de la tercera, y suavizándose las dos bandas de absorción cercanas a los 550nm. Las longitudes de onda de los máximos de las bandas de absorción son idénticas a las de la muestra de referencia (0h) por lo que no se señaló la presencia de ningún compuesto nuevo. No hay ninguna señal de absorción que pueda corresponderse con el nuevo pico cromatográfico que se empezó a identificar a 24h en las muestras del Grupo I. Quizá se debió a que la concentración del nuevo compuesto era inferior al límite de detección del espectrofotométrico empleado o que su espectro UV-Vis fuese similar al del compuesto inicial.

A partir de las $48 \mathrm{~h}$, las dos primeras bandas se unieron, presentado un máximo de absorción a $234 \mathrm{~nm}$, la tercera banda se mantuvo y desaparecieron las dos bandas de absorción detectada en la franja de los 550 $\mathrm{nm}$, lo que indicó que la tonalidad roja se había perdido por completo como ya se señaló con el análisis visual de las muestras. Desde las 72h de envejecimiento y hasta el final del proceso apareció una única banda de absorción muy ancha entre los 210 y los $320 \mathrm{~nm}$, desapareciendo el resto de las bandas.

Según los estudios de envejecimiento de F. Gosetti et col. [18], el colorante procedente de la cochinilla es generalmente mucho más foto-estable cuando está aplicado sobre un soporte que cuando está en disolución acuosa, conclusión que coincide con los resultados obtenidos en este estudio. La formación de los productos de degradación depende de la matriz en la que se encuentre, pero hasta ahora ningún autor 
ha podido identificarlos con certeza, simplemente se ha constatado la presencia de nuevos elementos como se ha señalado también durante este estudio.

\section{3.d. Amarillo de goma guta}

El color amarillo intenso obtenido de la resina de goma guta fue bastante resistente durante todo el proceso de envejecimiento, tanto en las muestras del Grupo I como del Grupo II (Fig. 1). La tonalidad amarilla permaneció hasta el final del ensayo de envejecimiento. Las muestras de referencia de los Grupos I y II mantuvieron la tonalidad original.

A pesar de que la tonalidad propia de este colorante permaneció bastante inalterable, los resultados obtenidos por el estudio cromatográfico y espectrofotométrico no revelaron que esta estabilidad fuese tan acusada (Fig. 7). Se observaron alteraciones del ácido gamboico (Fig. 2) y aparición de bastantes interferentes.

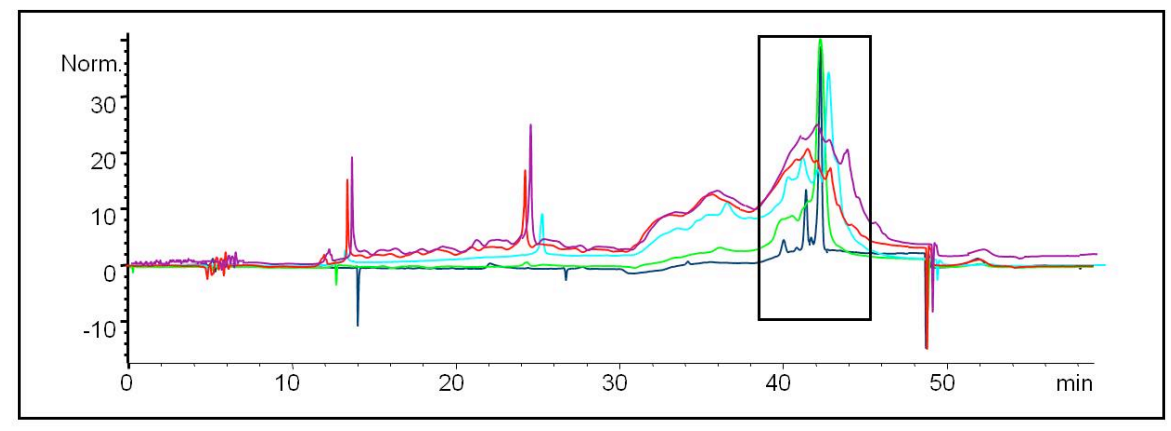

Cromatograma goma guta
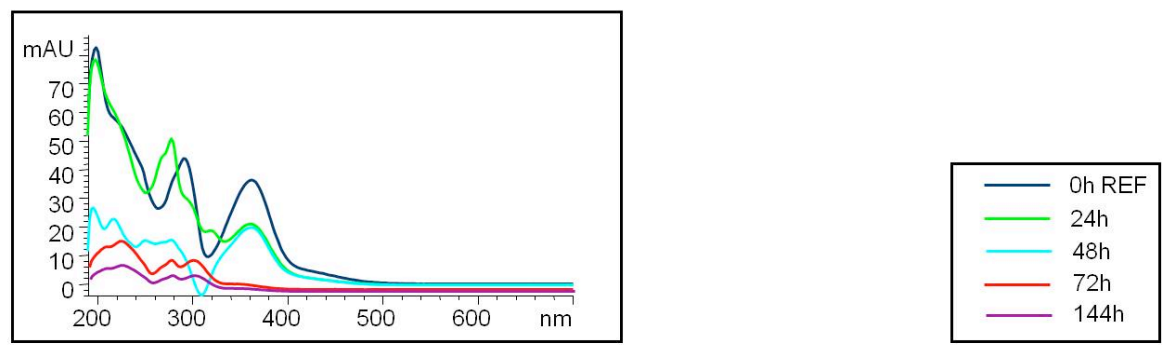

Espectro Uv-vis goma guta

Fig. 7. Cromatogramas y espectros UV-vis de la serie envejecida de amarillo de goma guta.

Los tres picos cromatográficos característicos de este colorante, con tR: 40,13, 41,83 y 42,37 min [16], se mantuvieron hasta las $48 \mathrm{~h}$ de envejecimiento. A este tiempo también aparece bastante bien reconocible un nuevo compuesto con $t_{R}: 24,01 \mathrm{~min}$. A partir de la muestra envejecida a $72 \mathrm{~h}$, las señales características del amarillo de goma guta se enmarañaron con multitud de picos solapados dando lugar a una "montaña" entre el min 31 y el min 46. El pico obtenido a las 24,01 min se hizo más evidente al igual que otro nuevo compuesto con $t_{\mathrm{R}}$ : 13,89 min. En el tramo final del proceso, estos dos picos de los nuevos compuestos ganaron en altura y área, y el deterioro de los picos característicos de este amarillo siguió en aumento.

En los espectros UV-vis realizados a las muestras del Grupo II (Fig. 7) se observó el mismo comportamiento que en las muestras analizadas por cromatografía del Grupo I. La muestra de referencia presentó 3 máximos de absorción a 205, 290 y 364 nm [16]. Esta última banda se corresponde con la absorción de las tonalidades amarillas. La muestra a $24 \mathrm{~h}$ resistió bastante bien las condiciones a las que estaba expuesta, aunque la segunda banda se desplazó hacia la izquierda $10 \mathrm{~nm}$. Aun así, el espectro era perfectamente reconocible. A las 48h, las dos primeras bandas de absorción desaparecieron permaneciendo la tercera a $364 \mathrm{~nm}$, siendo la principal y más característica de este colorante, por lo que también podría identificarse gracias a la permanencia de la tonalidad amarilla. Finalmente, a partir de las $72 \mathrm{~h}$ y hasta el final del proceso, las líneas de absorción desaparecen indicando la alteración completa del compuesto amarillo. 
En este caso los resultados no se pueden contrastar con estudios previos debido a la escasez de bibliografía referente a este compuesto colorante y su empleo en patrimonio. En todo caso se puede apuntar que los dos nuevos compuestos aparecidos serán de naturaleza posiblemente más polar que le ácido gamboico por presentar $t_{R}$ más reducido.

\section{Conclusiones}

El comportamiento ante el envejecimiento acelerado de los colorantes estudiados ha sido dispar, resultado esperable ya que sus composiciones son distintas, aunque sí se ha observado, como resultado común, y según reza en la bibliografía consultada, que los colorantes aplicados sobre un soporte celulósico adquieren mayor resistencia a las alteraciones provocadas por el envejecimiento acelerado [18, 15].

Se ha observado el efecto del envejecimiento acelerado gracias al control de tres parámetros: exposición a la luz, humedad relativa y temperatura. La combinación de los parámetros en condiciones de envejecimiento artificial permite simular la acción del paso del tiempo de manera acelerada en los materiales estudiados.

Se ha confirmado que estos materiales sí son sensibles a la exposición de estos parámetros, observándose cambios ópticos y químicos como consecuencia de las alteraciones, describiéndose con detalle durante la discusión de los resultados.

El rojo procedente de la raíz de Rubia tinctorum $L$. ha sido el colorante más estable al proceso de envejecimiento de entre todos los estudiados. Los colorantes azul de índigo, rojo carmín y amarillo de goma guta, además de alterarse, han dado lugar a la formación de nuevos compuestos. Estos nuevos compuestos no han podido ser caracterizados en este trabajo, pero abren una nueva vía de investigación que se desarrollará apoyada en este estudio preliminar y en la bibliografía consultada.

Las alteraciones de estos colorantes implican la imposibilidad de identificación en muchas ocasiones si no han estado conservados en las mejores condiciones de conservación. Esto implica que la "no identificación" de estos materiales en una obra de cierta antigüedad no supone su "no uso". Por lo tanto, no se podrá afirmar, de manera categórica, su ausencia. Afortunadamente, en algunos documentos gráficos las cubiertas han colaborado en la protección de los materiales, lo que ha permitido la conservación e identificación de estos colorantes orgánicos naturales.

Con este nuevo conocimiento, además de poder identificar los colorantes a pesar de encontrarse alterados, se puede establecer el estado de conservación en el que se encuentran y si el proceso de alteración ha comenzado.

\section{Agradecimientos}

Este trabajo fue realizado dentro del proyecto (MAT2014-58659-P) "Nuevas alternativas al conocimiento de los materiales y los procesos de conservación y restauración de obra gráfica y patrimonio documental" que está financiado por el Ministerio de Economía y Competitividad y por el Fondo Europeo de desarrollo regional FEDER. Así mismo A. López-Montes ha realizado este trabajo gracias a un contrato del programa "Ramón y Cajal" (RYC-2011) financiado por el Ministerio de Economía y Competitividad. 